\title{
Impact of the SARS-Cov-2 Virus Pandemic on Children and Adults with Congenital Heart Disease: Its Burden and Risk Factors
}

\author{
John Jairo Araujo, MD ${ }^{1,2^{*}}$ (D) \\ ${ }^{1}$ Cardiologist-Echocardilogist, Department of Pediatric and Adult Congenital Heart Disease, Somer in \\ Care Cardiovascular Center, Colombia \\ ${ }^{2}$ Cochair Adult Congenital Heart Disease Council, Inter American Society of Cardiology, Colombia
}

*Corresponding author: John Jairo Araujo, MD, Cardiologist-Echocardilogist, Departament of Pediatric and Adult Congenital Heart Disease; Cochair Adult Congenital Heart Disease Council, Inter American Society of Cardiology, Rionegro, 054040, Colombia, Tel: +5745624020

\begin{abstract}
The novel coronavirus causes severe acute respiratory syndrome 2, and can infect any population. With the currently available information on the behavior of the pandemic, we conclude that the virus has come to stay. It is clear that the most aggressive behavior and worst outcome of the infection occurs in already established risk groups, such as those with cardiovascular disease. Those with congenital heart disease make up a special risk group. Due to this group's high heterogeneity and the paucity of published cases related to coronavirus infection, its ultimate behavior cannot yet be known. Based on the anatomical and hemodynamic abnormalities with systemic repercussions which accompany congenital heart disease, this review was carried out with the aim of describing and analyzing this population's risk factors and potentially increased vulnerability to coronavirus infection. Systemic hematological abnormalities, along with the prothrombotic state, proinflammatory state, and altered immune response in cyanotic congenital heart disease are highlighted; along with the arrhythmias, pulmonary hypertension, underlying heart failure and target organ damage, among others, present in unrepaired congenital heart disease. This review searched for articles in PubMed, Science Direct, SciELO, MD Consult and Google Scholar using the MeSH terms "coronavirus disease 2019", "COVID-19", "SARS-CoV-2 pandemic", "coronavirus and cardiovascular system", "congenital heart diseases", "hematologic disorders in congenital heart disease", and "pro-inflammatory mediators". Finally, a brief description of the collateral effects of the pandemic on the care of this population is provided.
\end{abstract}

\section{Keywords}

SARS-CoV-2 virus, Congenital heart disease, Coronavirus pandemic, Pro-inflammatory mediators

\section{Introduction}

The novel coronavirus causes severe acute respiratory syndrome 2 (SARS-CoV-2) and its disease has been called COVID-19 (Coronavirus Disease 2019). The epidemic outbreak began in December 2019 in the city of Wuhan (China), detected in patients who developed a severe form of pulmonary infection and respiratory distress. It was declared a pandemic by the World Health Organization (WHO) in March 2020 [1,2]. From the beginning of the epidemic to date, several articles have been published on cardiovascular risk factors and their relationship to coronavirus infection [3-5]. With regard to congenital heart disease (CHD), Sabatino J, et al. reported the results of a nationwide survey conducted at various CHD centers in Italy. The study included 76 COVID-19 infected CHD cases recorded between February 21 and April 4, 2020, including four children (under the age of 18) and 72 adults. The complications shown were: heart failure (HF) (9\%), palpitations/arrhythmias (3\%), stroke/transient ischemic attack (3\%), and pulmonary hypertension (PH) (3\%). Cardiovascular symptoms such as chest pain (1\%), myocardial injury (1\%) and pericardial effusion (1\%) were also recorded. They observed a mild COVID-19 clinical course in their cohort of CHD patients [6]. Isolated cases showing the behavior of the infection in complex CHDs have also been published [7]. Likewise, another recent publication attempted to stratify the $\mathrm{CHD}$ risk due to the coronavirus [8]. However, even with this information, it is not possible to calculate the actual impact of the COVID-19 pandemic on the pediatric and adult CHD population.

Citation: Araujo JJ (2020) Impact of the SARS-Cov-2 Virus Pandemic on Children and Adults with Congenital Heart Disease: Its Burden and Risk Factors. Int J Clin Cardiol 7:190. doi.org/10.23937/23782951/1410190

Accepted: July 23, 2020; Published: July 25, 2020

Copyright: (C) 2020 Araujo JJ. This is an open-access article distributed under the terms of the Creative Commons Attribution License, which permits unrestricted use, distribution, and reproduction in any medium, provided the original author and source are credited. 
Previous experiences with the behavior of epidemics such as H1N1 in connection with CHDs could guide us with regard to the risk factors which increase unfavorable outcomes. A study performed at the National Institute of Child on 74 children with pneumonia during the AH1N1/2009 influenza pandemic showed a statistical association with mortality and the development of nosocomial pneumonia. The most frequent contributing factors were malnutrition, respiratory infections, CHD and neurological deficits [9]. A study in Beijing (China) on the behavior of influenza from 2007 to 2013 attributed an average of 2,375 deaths to influenza per season, accounting for $3 \%$ of all deaths. Overall, $81 \%$ of the deaths attributed to influenza occurred in adults aged $\geq 65$ years, and the influenza-associated mortality rate in this age group was higher than the rate among those aged $<65$ years $(P<0.05)$. The mortality rate associated with the 2009 H1N1 pandemic in 2009/2010 was comparable to that of seasonal influenza during the seasonal years. People aged $<65$ years represented a greater proportion of all deaths during the influenza $A$ (H1N1) pandemic period than during the seasonal epidemics $(P<0.05)[10]$.

For the current SARS-CoV-2 virus pandemic, we still cannot conclude what its behavior will be. So far, the containment measures applied have tried to mitigate its spread, especially among risk groups, including those with CHD. This may perhaps be a contributing factor to our not really knowing what the development will be in this population. The objective of this paper is to describe, generally, the main risk factors related to repaired and unrepaired CHDs and their relationship to a potential coronavirus infection. At the end, some of the pandemic's collateral effects on the care of this population are described.

\section{Methods}

A narrative and analytical review was conducted to answer the question of which CHD risk factors could increase vulnerability to the coronavirus infection. A search of the PubMed, Science Direct, SciELO, MD Consult and Google Scholar databases was performed, using the MeSH terms "coronavirus disease 2019", "COVID-19", "SARS-CoV-2 pandemic", "coronavirus and cardiovascular system", "congenital heart diseases", "hematologic disorders in congenital heart disease", and "pro-inflammatory mediators". Altogether, 43 articles in English and 2 in Spanish were included, along with two internet data sources (accessed on June 5, 2020). Articles containing related information were selected. Eligible articles were screened and the titles and abstracts of all the articles found were reviewed. Eight were discarded because they had no direct relationship to the topic. In the end, 39 articles were included as the most relevant (Figure 1).

\section{Discussion}

\section{Living with the SARS-CoV-2 virus}

The SARS-CoV-2 virus is derived from other coronavirus genera such as SARS-CoV1 and MERS corona-

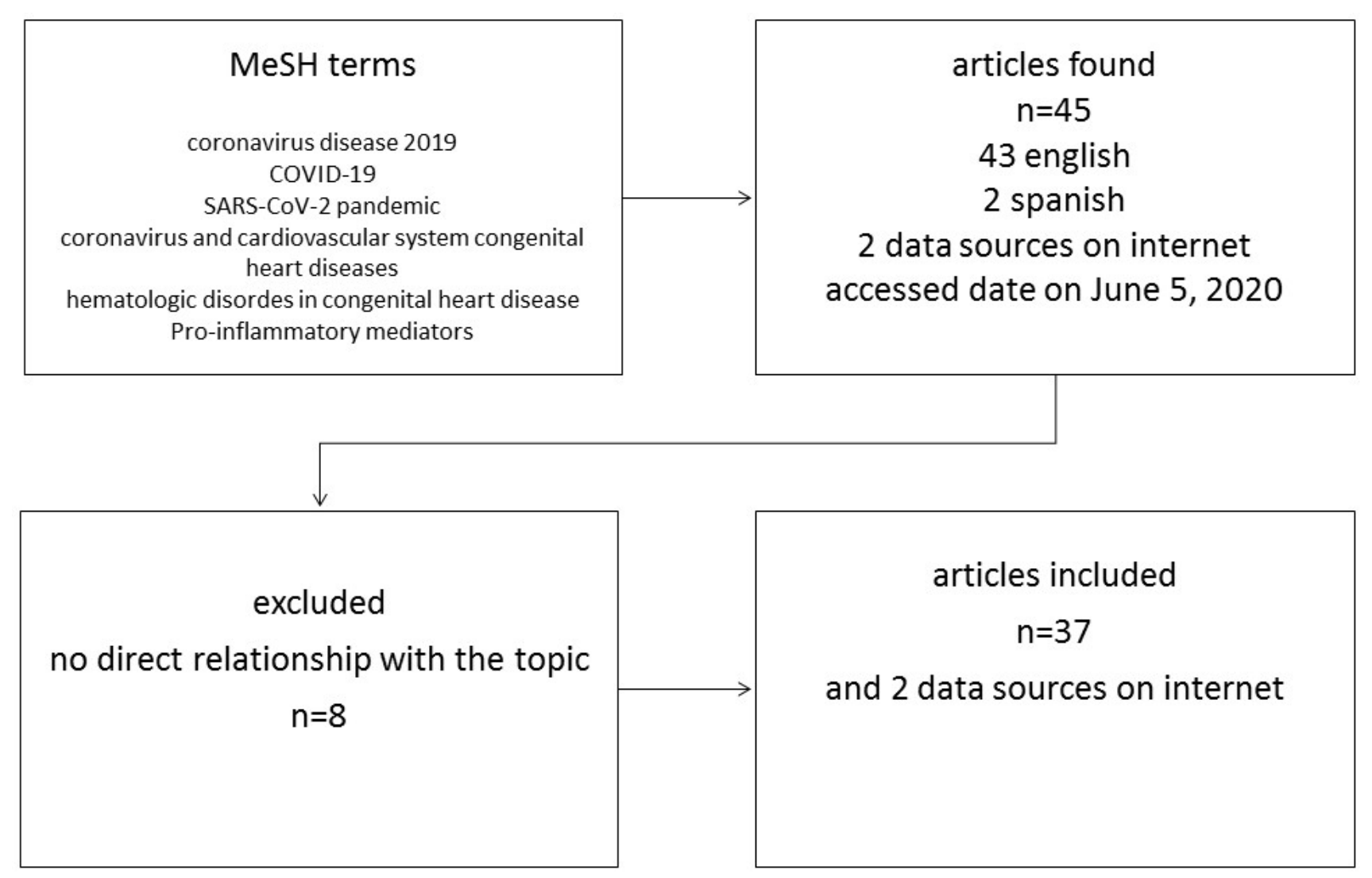

Figure 1: Identification process and selection of articles. 
virus. These cause severe respiratory infections every year with a fatality rate which has remained constant at 9-36\% [11]. Other human coronaviruses include HCoVOC43 and HCoV-HKU1, which cause outbreaks of respiratory infection during winter seasons $[12,13]$. The SARS-CoV-2 virus may stabilize in summer and spring, but can increase its proliferation and transmission in fall and winter. Winter coincides with the influenza peak, suggesting that transmission and infection are facilitated by season changes. The lethality of the SARSC-CoV-2 virus is $0.6-3.5 \%$ [14]; compared to the SARS-CoV1 virus, this seems like a low lethality, but it is high compared to the lethality of HCoV-OC43 and HCoV-HKU1. Overall, the seasonal variation led to a lower epidemic peak during the pandemic, but higher and more severe seasonal outbreaks are to be expected. So far, there is no permanent immunity, and therefore it is to be expected that the SARS-COV-2 virus will recirculate and have cycles of infection, as occurs with the influenza virus and other human beta coronaviruses [15].

Learning to live with and adapt to this new situation is important for restarting all the healthcare activities that have been reduced in many countries due to the pandemic. The purpose of all the containment measures applied was to decrease the intensity of the epidemic peak (not eliminate contagion or infection), thus reducing the risk of healthcare system saturation and gaining time for adaptations and the development of possible vaccines and effective treatments. In several countries, lethality was increased by the saturation of intensive care units and respirators. Social distancing worked in China, eliminating $50-60 \%$ of cases of infection, and mitigating the possibility of resurgence [16]. Understanding that the SARS-CoV-2 virus has arrived to stay is crucial for learning to live with the enemy. Based on this premise, many European and some Latin Amer- ican countries have gradually restarted their activities. However, at the time of this writing, we do not know what the behavior and resurgence of the SARS-CoV-2 virus will be over the next few months. The experts state that if immunity is permanent and lasting, the projected disappearance of the virus will be in five years. Therefore, the containment measures will need to be applied at least until 2022 [15].

\section{Overview of the burden of congenital heart dis- ease and vulnerability}

According to the epidemiological reports known to date, the SARS-CoV-2 virus has had a greater impact on people over the age of 60 with cardiovascular (systemic arterial hypertension [SAH], HF), pulmonary (chronic obstructive pulmonary disease, asthma, pulmonary hypertension $[\mathrm{PH}]$ ) and systemic (diabetes, cancer) comorbidities. However, as the pandemic spread, cases of young people and children without apparent risk factors began to be reported. This indicates that the SARSCoV-2 virus can affect anybody, regardless of age, sex, race, social condition and health $[17,18]$.

Various cardiovascular, respiratory and systemic abnormalities converge in complex CHD cases, even if they have already been repaired. Congenital heart disease residuals and sequelae, manifested as atrial septal defects (ASDs) or ventricular septal defects (VSDs), valvular regurgitation and arrhythmias, among others, predispose to hemodynamic abnormalities with systemic repercussions affecting cardiovascular homeostasis to the point where any noxa will destabilize the individual, with serious consequences (Figure 2).

\section{Anatomical, hemodynamic and systemic abnor- malities which could have an increased risk}

Interatrial or interventricular septal defects: Those

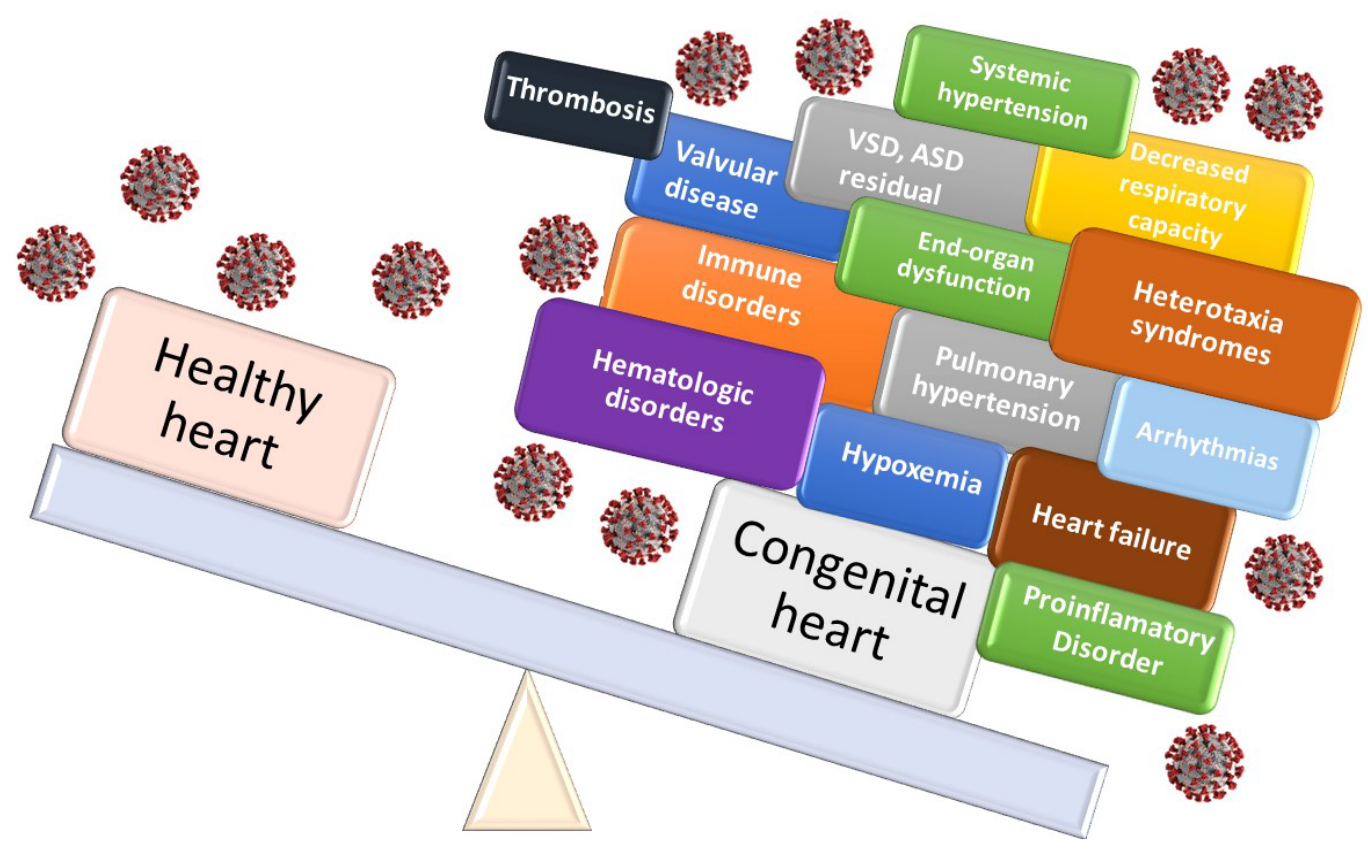

Figure 2: Congenital heart disease burden and risk with coronavirus infection. 
that are large or medium-sized (unrepaired) or residual defects with hemodynamically significant shunting (QP: QS > 1.5 [pulmonary blood flow: Systemic blood flow, respectively]). They cause increased pulmonary flow, vascular congestion, heart chamber dilation and $\mathrm{PH}$. Thousands of children around the world die each year due to complicated respiratory infections caused by influenza virus, adenovirus, respiratory syncytial virus or bacteria which include Pneumococcus, Staphylococcus, Klebsiella pneumoniae and Mycoplasma pneumoniae, among the most frequent. Often, coupled with these respiratory deaths is an associated CHD [19].

A study in Denmark of 1,168 adults diagnosed with ASDs from 1977 to 2009 compared their risk of hospitalization for pneumonia, use of antibiotics, and mortality to that of age and gender-matched general population controls. The researchers showed that patients with both closed $(n=863)$ and unclosed $(n=305)$ ASDs had a higher risk of hospitalization for pneumonia (adjusted hazard ratios [HRs] 2.8, 95\% confidence interval $[\mathrm{Cl}] 2.3$ to 3.5 ; and $3.1,95 \% \mathrm{Cl} 2.4$ to 4.0 , respectively) than controls. Patients with ASDs also used more antibiotics than the controls [20].

We know that the SARS-CoV-2 virus can cause severe necrotizing pneumonias which, undoubtedly, when combined with a CHD with hemodynamic repercussions, could lead to worse outcomes.

Pulmonary hypertension: Unrepaired CHDs, or those repaired late, in adulthood, cause PH. Patients with large defects, all those with truncus arteriosus, $50 \%$ of those with VSDs and $10 \%$ of those with ASDs will develop PH. For patients with ASDs, the incidence of PH is greater in those with sinus venosus defects (16\%) than in those with ostium secundum defects (4\%). In adults, the estimated prevalence of $\mathrm{PH}$ associated with unrepaired CHD ranges from 1.6 to 12.5 cases per million adults, with $25-50 \%$ of this population affected by the most extreme form, Eisenmenger syndrome [21-23].

Pulmonary hypertension is seriously destabilized by respiratory infections. The acute rise in airway resistance caused by inflammation worsens the degree of $\mathrm{PH}$. As a result, respiratory deterioration impacts on cardiovascular effort, especially that of the right chambers, which often collapses in failure [24]. At the moment, there is no strong evidence regarding the behavior of COVID-19 infection in patients with $\mathrm{PH}$. However, a collateral effect of the pandemic is the poor follow-up of the disease due to confinement measures. A recent report in China showed that nearly $70 \%$ of the participants in a survey experienced a shortage of medications during the COVID-19 outbreak. Altogether, $24.2 \%$ of patients indicated that medications were discontinued due to an insufficient supply [25]. With the paucity of information to date, we still do not know what the outcome will be in this population.
Valvular disease: Present in repaired and unrepaired $\mathrm{CHDs}$. These are a very common residual and sequela following repair of various heart defects. Some of the most common ones include pulmonary regurgitation (PR) following tetralogy of Fallot (TF) or congenital pulmonary stenosis repair, aortic regurgitation in aortic valvuloplasty due to congenital stenosis, and tricuspid regurgitation in Ebstein's anomaly. Many of these residual lesions are well tolerated under baseline conditions and are followed for years before definitive repair $[26,27]$. However, they are not immune to complications caused by pulmonary infections, with increased symptoms and hemodynamic repercussions under situations of stress and increased cardiac effort. This would also be the case for unrepaired or residual valvular stenosis. Currently, there is no information on SARS-CoV-2 infection and potential hemodynamic problems for children and adults. However, it may be assumed that the general repercussions on ventricular function and pulmonary pressure could be serious.

Arrhythmias: These are common after CHD repair; more than $80 \%$ of adults with CHD are known to develop some type of arrhythmia. The most common arrhythmia is atrial fibrillation in $86 \%$ of cases, followed by atrial flutter $20 \%$, ventricular tachycardia $5-7 \%$, symptomatic bradycardia 3-5\%, and pacemaker malfunction $5 \%[28,29]$. Up to $36 \%$ of Ebstein's anomaly cases also have pre-excitation syndrome [30]. In both children and adults, the CHDs that cause the most arrhythmias are classical and congenitally corrected transposition of the great arteries, tetralogy of Fallot, Ebstein's anomaly and single ventricle [31]. Serious, persistent arrhythmias negatively affect cardiac function. They cause asynchrony between atrial and ventricular contraction, affecting ventricular filling and cardiac output. When the heart chambers are dilated, they predispose to thrombus formation and secondary emboli, especially in adults. Recent reports have documented that the SARS-CoV-2 virus was associated with arrhythmias in $16.7 \%$ of a cohort of 138 patients in a Chinese hospital [32]. It is assumed that with the arrhythmogenic substrate of repaired or unrepaired CHDs, the incidence of serious arrhythmias could be greater and more deleterious. Children and adults with $\mathrm{CHD}$ also have various electrolyte disturbances $(\mathrm{K}, \mathrm{Mg}, \mathrm{Ca})$ caused by the chronic administration of diuretics which, together with medications such as chloroquine or macrolides (which have been used to treat SARS-CoV-2 viral infections), may prolong the QTc interval (corrected QT interval) and lead to serious arrhythmias [33-35].

In addition, it should be highlighted that, so far, the various clinical and experimental studies administering chloroquine alone or combined with a macrolide to treat SARS-CoV-2 viral infection have shown no benefit [36]. On the contrary, the arrhythmic complications have been so frequent that support for their use has been withdrawn and the clinical trials with this medica- 
tion have been discontinued [37].

Hypoxemia: Manifested by decreased arterial oxygen saturation to $<90 \%$ on room air. It is considered to be severe when $<85 \%$. This is a consequence of unrepaired cyanotic CHDs, persistent venous-arterial shunts, low pulmonary flow CHDs, $\mathrm{PH}$ and Eisenmenger syndrome [22].

Oxygen free radicals produced during aerobic metabolism, including hydroxyl $(\mathrm{OH})$ radicals, superoxide anions $\left(\mathrm{O}_{2}\right)$ and hydrogen peroxide $\left(\mathrm{H}_{2} \mathrm{O}_{2}\right)$ are essential in many processes, such as in the intracellular activation system, immunity and the defense against microorganisms. With lower arterial oxygen concentrations, the oxidative chain and inflammatory response to external pathogens is diminished, favoring the multiplication of bacteria and anaerobic germs [38,39].

One characteristic of SARS-CoV-2 viral infection is persistent hypoxemia, "happy hypoxia"; it has been shown that, in the initial stages of the infection, patients experience severe desaturation without respiratory distress [40]. An inflammatory reaction in the pulmonary blood vessel network could trigger a protein cascade which would stimulate coagulation and prevent adequate blood oxygenation. Early hypoxia triggers severe pneumonia and death $[41,42]$.

In patients with CHDs and baseline hypoxemia, the outcome is still unknown; however, it is assumed that, based on what has been explained, it could be very deleterious (Figure 3).

Heart failure: This frequently has a multifactorial etiology, as occurs in many CHDs. The fragile reserve to respond to hemodynamic stress caused by a noxa leaves this population at high risk of cardiovascular collapse, especially those with repaired or unrepaired univentricular heart physiology. Zhou, et al. found HF in $23 \%$ of patients hospitalized for SARS-CoV-2 virus infection [43]. Heart failure becomes even more significant in CHDs with a univentricular heart physiology, especially those with right heart morphology.

Systemic arterial hypertension: Secondary and present in children and adults with repaired or unrepaired CHDs accompanied by obstructions of the aortic arch, mitral valve or aortic valve. Primary essential forms of $\mathrm{SAH}$ are common in adults after the fourth decade of life. Recent data from the pandemic have shown the virus's affinity for angiotensin-converting enzyme 2 (ACE2), suggesting that treatment with ACE inhibitors (angiotensin-converting enzyme inhibitors) and/or angiotensin II receptor blockers (ARBs) could be a risk factor for serious illness in hospitalized patients with coronavirus infection. This initial disinformation and lack of knowledge led many patients to discontinue their medications. This behavior, assumed in part by some physicians, had the immediate consequence of worsening the CHDs and increasing $\mathrm{HF}$ and $\mathrm{SAH}$, and, to a lesser extent, producing and increasing postcapillary $\mathrm{PH}$. The SARS-CoV-2 virus could increase its lethality in patients with these hemodynamic conditions. Thus, it is recommended that these medications not be discontinued, due to a lack of clinical or scientific evidence [44].

Immune disorders: Various conotruncal CHDs such as TF, double-outlet right ventricle and truncus arteriosus. They are usually accompanied by $22 q 11$ deletion syndrome and are characterized by the clinical triad of CHD, hypocalcemia and primary immunodeficien-

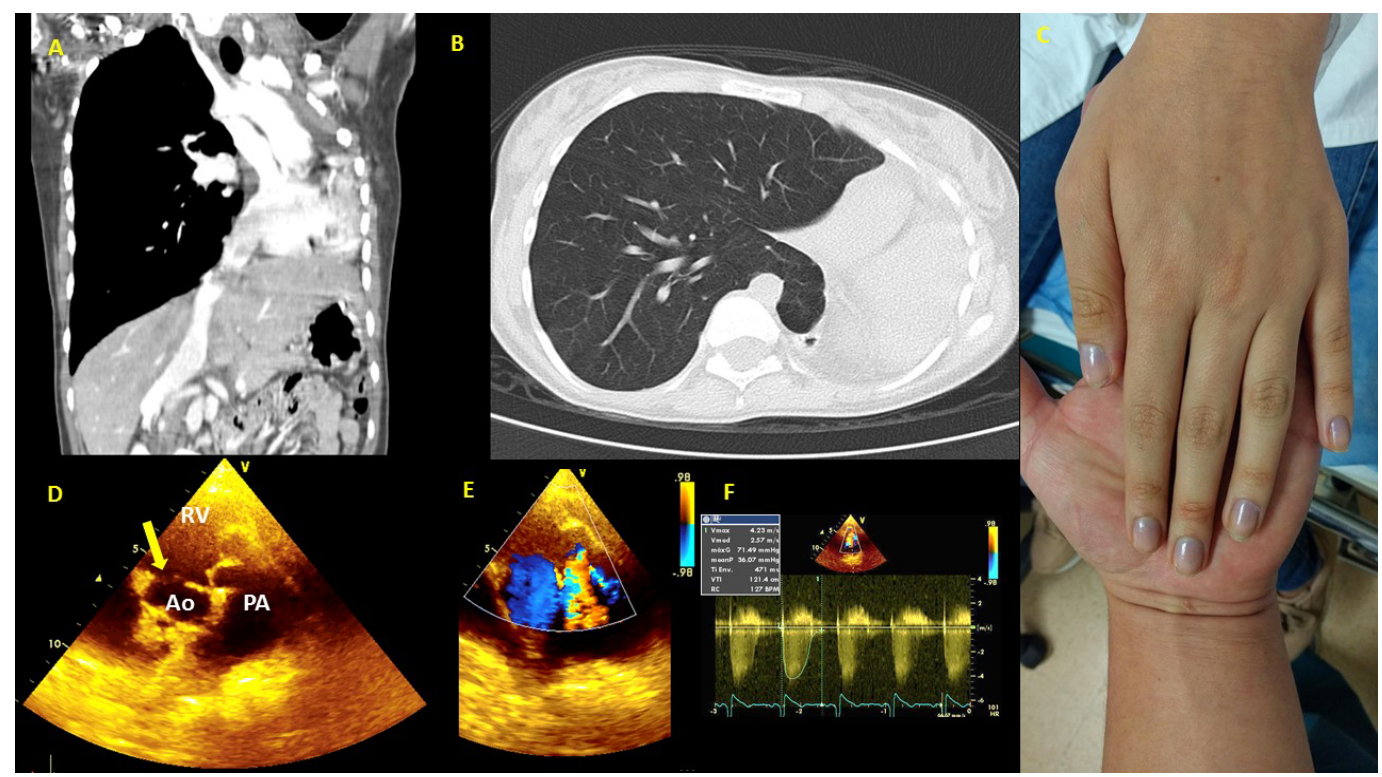

Figure 3: 16-year-old female with severe unrepaired CHD, multiple risk factors at the same time. (A) CT (Computed Tomography) thorax: Left lung agenesis, and important scoliosis; (B) CT thorax: Right lung hyperinflation compensatory, and herniation to left hemithorax; $(C)$ Left hand showing marked central hypoxemia due to unrepaired severe $\mathrm{CHD}$, saturation $75 \%$ on room air; (D) Echocardiography study: Short axis view shows double-outlet RV. Yellow arrow shows relationship between the VSD and the blood vessels; (E) The same view in D, doppler color shows pulmonary valve stenosis; (F) Doppler in pulmonary valve with $71 \mathrm{mmHg}$ peak gradient. 
cy. Thymic hypoplasia or aplasia causes lymphocyte subpopulation disorders. Flow cytometric peripheral blood counts of T lymphocytes, B lymphocytes, NKs and monocytes often reveal altered numbers and function [45]. It is understandable that the immune response to a noxa such as the SARS-CoV-2 virus should behave aggressively in this patient population, but at the moment, this is only a supposition.

Proinflammatory state in congenital heart disease: Cyanotic and acyanotic CHDs have an increased expression of cytokines like TNF- $\alpha$ and IL-6. Yilmaz E, et al. demonstrated the relationship between ghrelin (a 28-amino-acid peptide which is a potent stimulator of growth hormone release that has been implicated in the control of food intake and energy homeostasis in humans) levels in CHD and TNF- $\alpha$ and IL-6. They measured serum ghrelin, TNF- $\alpha$ and IL- 6 levels in 68 patients (47 acyanotic and 21 cyanotic CHD patients) and in 25 control subjects. In comparison to controls, serum ghrelin and TNF- $\alpha$ levels were significantly higher in acyanotic and cyanotic CHD patients $(P<0.0001)$. In acyanotic and cyanotic patients, there was a positive correlation between ghrelin and TNF- $\alpha(r=0.485, P<0.05$ and $r=0.573, P<0.01$, respectively). The relationship between ghrelin and the cytokines may be explained by the possible effect of chronic congestive heart failure and chronic shunt hypoxemia [46]. Other studies have shown growing evidence that the level of anti-inflammatory cytokines in the blood has a remarkably positive association with CHD [47].

With regard to the proinflammatory state in CHDs, we do not know for sure what its behavior would be in a SARS-CoV-2 virus infection. It is likely that the described altered expression of cytokines would play a role in the immune response and the clinical phenotype. However, at the moment we do not have available data to support this. Even so, it is important to understand that for clinical interpretation purposes, CHD patients have baseline cytokine level alterations.

COVID-19 infection and its association with Kawasaki disease: International data suggest the development of a proinflammatory syndrome with features of Kawasaki disease (KD) or toxic shock syndrome (TSS) in children, possibly related to COVID-19 [48]. In the past 20 years, viruses from the coronavirus family have been proposed as possibly implicated in the pathogenesis of KD. In 2005, a group of researchers in the United States (US) identified a novel human coronavirus, designated New Haven coronavirus ( $\mathrm{HCOV}-\mathrm{NH}$ ), in the respiratory secretions of eight of 11 children with KD, versus one of 22 controls tested by RT-PCR (reverse transcription polymerase chain reaction) [49]. The symptoms described are fever, gastrointestinal symptoms, rash, conjunctivitis, and lip redness/swelling. The proinflammatory effect of SARS-CoV-2 has been reported in adults with the most severe respiratory complications of COVID-19.
Many of these patients have a constellation of features classified under the term cytokine storm, such as fever, lymphopenia, elevated transaminases, lactate dehydrogenase, D-dimer, and ferritin, in keeping with TSS. Likewise, TSS is a form of cytokine storm, and might affect patients with KD [50].

The report of cases seen in the Province of Bergamo following the SARS-CoV-2 epidemic showed an increased monthly incidence of KD 30 times higher than that of the previous five years. Among patients infected with the coronavirus there were elevated levels of IL-2R, IL-18 and CXCL 9 cytokines, and mildly increased IFN- $\gamma$ and IL-8. The observed pattern of cytokine expression suggests an interferon signaling component, along with IL- 6 and IL-10 production, seen in KD [51]. The systemic inflammatory response described in CHDs could possibly have an as yet unknown behavior. Prospective studies would need to be carried out in this population.

Hematological disorders: Cyanotic CHDs are accompanied by various hematologic disorders, the most common being $[52,53]$.

a) Thrombocytopenia, seen in CHDs with large persistent septal defects due to which circulating megakaryocytes do not travel from the systemic venous circulation to the lungs, where they are degraded to mature platelet forms. Rather, they are detoured to the systemic arterial circulation by the defects, and the number of circulating platelets is low.

b) Secondary erythrocytosis (SE), a consequence of hypoxemia and $\mathrm{PH}$ in unrepaired $\mathrm{CHDs}$ with persistent venous-arterial shunts. The increased number of erythrocytes increases blood viscosity, consequently slowing blood flow between the dense capillary vessels. This would become very significant in a SARS-CoV-2 infection where hypoxemia + $\mathrm{PH}+$ hyperviscosity + pulmonary capillary thrombosis converge, this could increase the infection's lethality.

c) Large or peripheral vessel thrombosis: Caused by blood hyperviscosity in the context of a high hematocrit and hemoglobin $(\mathrm{Hb})$ due to SE. It is assumed that SARSCoV-2 causes alveolar damage, followed by an inflammatory reaction and microvascular pulmonary thrombosis. This progressive endothelial thromboinflammatory syndrome may develop in the microvasculature of all organs and systems. Hospitalized patients with moderate and severe COVID-19 and those with poorer outcomes are noted to have prolonged prothrombin time, elevated $\mathrm{D}$ dimer, and activated partial thromboplastin time. In the context of a clinical picture that is consistent with disseminated intravascular thrombosis, it is reasonable to speculate that COVID-19 would be associated with venous or arterial thrombi; however, the incidence has not been published [54].

d) Iron deficiency is present in various CHDs, especially cyanotic ones. Iron is essential for $\mathrm{Hb}$ synthesis 
and oxygen transport. SARS-CoV-2 infection causes an inflammatory reaction in the pulmonary blood vessel network and triggers a protein cascade which stimulates coagulation and prevents proper blood oxygenation. If this occurs with low Hb levels, it is assumed that hypoxemia could be greater and more deleterious in the CHD patient population.

Decreased functional respiratory capacity: Occurs as a result of age and various mechanisms related to the hemodynamic repercussions of CHDs. Patients with univentricular physiology with Fontan circulation tend to have a decreased ventilatory capacity and lymphatic drainage of the airways due to increased circulatory hydrostatic pressure, causing varying degrees of pulmonary congestion at rest which may triple during pulmonary infection. The bronchi and small airways have increased resistance, making them susceptible to respiratory complications. Complications such as plastic bronchitis further increase airway resistance. Mechanical complications such as diaphragmatic paralysis, which tends to occur in some surgical repairs of congenital heart defects, place this population at a disadvantage in an episode of respiratory distress.

End-organ dysfunction: Liver dysfunction in patients with Fontan circulation has greater than $40 \%$ prevalence at 30 years of age [55]. Cyanotic CHDs cause hypoperfusion and hypoxia in organs such as the kidneys, brain, heart and liver. The progressive development of fibrosis in these organs irreversibly deteriorates their function. There is clinical and/or laboratory evidence of end-organ dysfunction in many complex CHDs.

Renal injury estimates vary as to the incidence of acute kidney injury in COVID-19, ranging from 0.5\% to $15 \%$ [56]. Among hospitalized patients, the rates of proteinuria $(43.9 \%)$ and hematuria $(26.7 \%)$ appear to be even higher [57]. Renal injury occurs in the first few days after admission in patients with baseline chronic kidney disease, and after 7 to 10 days in patients with normal baseline renal function. Mechanisms of renal injury have been hypothesized to include acute tubular necrosis, direct cytotoxic effects of the virus itself, and immune-mediated damage [58].

Other research has shown liver injury in COVID-19, manifested with transaminitis, with an incidence of $21 \%$ to $37 \%$, and as high as $48 \%$ to $62 \%$ in patients who are critically ill or who do not survive [45].

Heterotaxy syndromes: Occurring in just over 1 in 10,000 live births, the associated CHDs are always severe [59]. They are accompanied by various systemic abnormalities, and immunological disorders are significant. Recent retrospective studies have found sepsis in almost $20 \%$ of patients over two-years-old, and this is nearly twice the rate of infection in children with comparable heart disease but without heterotaxy [60]. If hospitalized, they have a greater risk of nosocomial sepsis. Asplenic and even polysplenic patients are at a serious disadvantage in infection. It is anticipated that SARS-CoV-2 viral infection could be very lethal in this population.

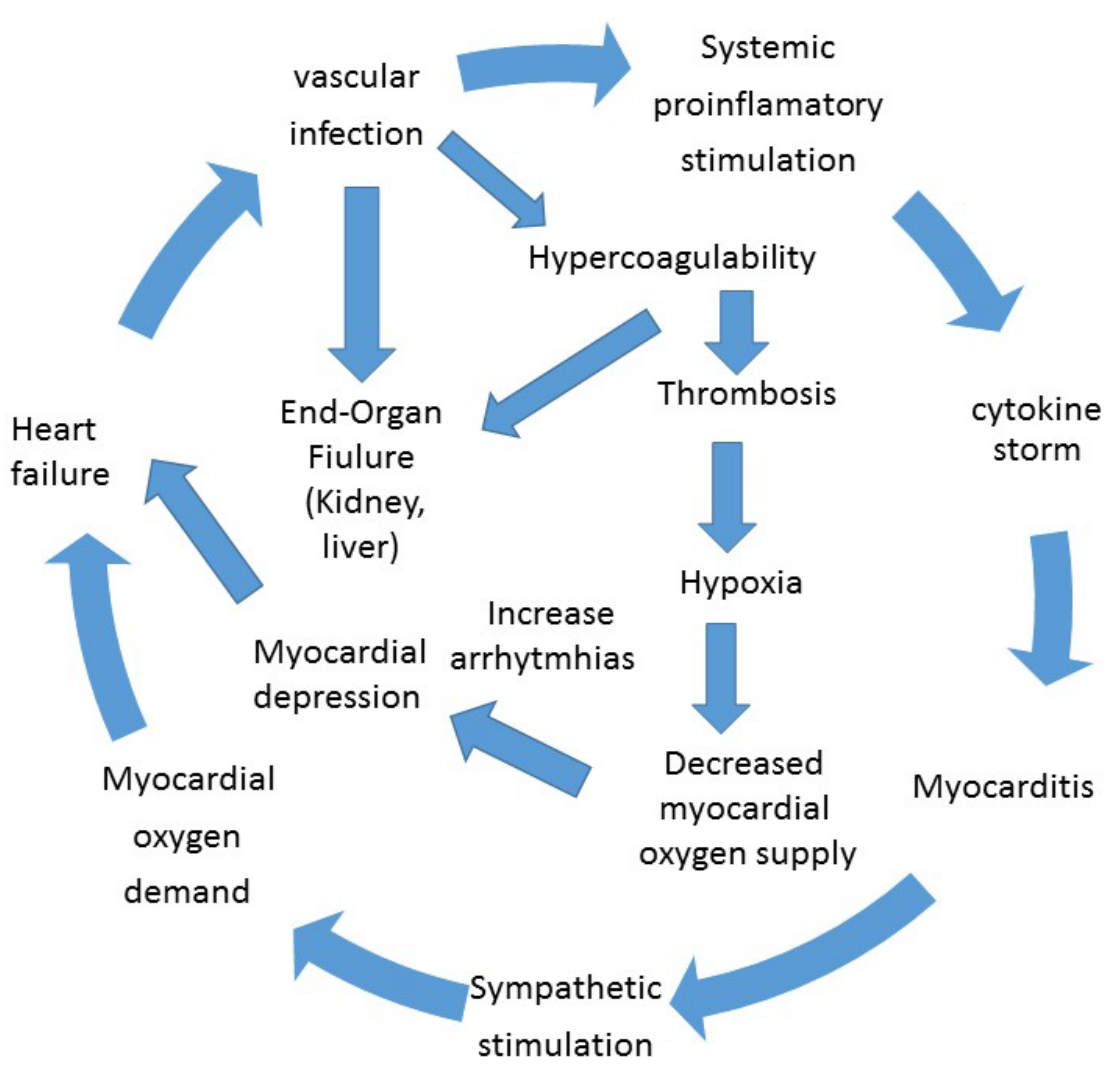

Figure 4: Possible damage mechanisms on cardiovascular system. 
Figure 4 summarizes the possible damage mechanisms on cardiovascular system.

\section{Collateral effects of the SARS-CoV-2 virus pandem- ic on the specialized care of children and adults with congenital heart disease}

The SARS-CoV-2 virus pandemic changed the world, and the manner in which we were carrying out specialized medical care was dramatically affected. A variety of reasons explain this change, principally:

a) Fear of consulting and being infected with the SARSCoV-2 virus: Without a doubt, this is the main reason why children and adults have not attended specialized medical care. Lack of knowledge has filled patients and physicians with fear. It is understood that the virus may be asymptomatic for at least seven days. The concentration of several people within less than two meters of each other in an enclosed space, such as a hospital or doctor's office waiting room, facilitates viral spread. Adults minimize their symptoms. Signs and symptoms such as tachycardia, diaphoresis, weakness or dizziness are often deemed "normal" or secondary to worry about the pandemic. This "masking" phenomenon is simply the manifestation of CHD decompensation. Delayed care may lead to serious consequences.

b) Changes in local and national health policies: in all affected countries, the policies and priorities for economic and logistical resources were assigned to the pandemic. Therefore, all other diseases, including cardiovascular diseases, became secondary.

c) Closure or significant decrease in outpatient care services: the healthcare policies adopted by the vast majority of clinics and hospitals completely closed all outpatient hospital services in order to prevent crowding in enclosed spaces. Consequently, only emergency and intensive care services remained active.

d) Infection of healthcare personnel responsible for the care of children and adults with CHDs: A consequence of overexposure to the virus from providing care in hospitals and clinics during the pandemic. Official data from the Spanish Ministry of Health confirm that three out of ten infected people were healthcare workers, reaching 43,000 from the beginning of the pandemic in February to June [61].

e) Transformation of specialized hospitals into COVID hospitals: In most countries, the best specialized hospitals, including cardiovascular centers, tend to have the best infrastructure and supply of resources. The number of beds, personnel, operating rooms, intensive care units and healthcare personnel tends to be large. The policies adopted by healthcare authorities made these centers reference hospitals for patients infected with the SARS-CoV-2 virus, now calling them "COVID hospitals". It is reasonable to understand that the care of children and adults with CHD has been relegated to second place, at least until the pandemic passes; and, based on the data we have so far, this is still far away for many countries.

f) Decreased patient and family financial resources: due to the containment and confinement measures, many adults have had to stop working. The direct consequence has been the loss of jobs and reduction of financial resources [62]. As a collateral effect, these people, who should be economically productive, are no longer contributing resources to the healthcare system. They are now unemployed and lack healthcare coverage. We should be prepared for an avalanche of decompensated CHD patients in the following months.

g) Changes in the priorities of educational activities: the cardiology and $\mathrm{CHD}$ fellow training programs are undergoing transformations worldwide. In the United States, the Accreditation Council for Graduate Medical Education (ACGME) is allowing institutions to self-declare Pandemic Emergency Status. The fellows-in-training are learning about cardiovascular manifestations of the SARS-CoV-2 virus, including myocardial injury, myopericarditis, heart failure, arrhythmias, and thrombophilias in real time and through international reports [63]. Meanwhile, onsite CHD training is currently reduced to only online attendance. The consequence may be fewer specialists to renew the slots of those who have finished their time. There could also be losses to follow up due to a lack of transition from pediatric cardiology to adult CHD care.

h) Mental burden and stress of healthcare personnel, along with personnel losses: Healthcare professionals experience feelings of extreme vulnerability, uncertainty and threat to their lives, together with somatic and cognitive symptoms of anxiety. The pressure to act quickly and diagnose, isolate and successfully treat has been overwhelming, especially in the midst of intense public and media scrutiny. These individuals have been under greater psychological pressure, experiencing high rates of psychiatric morbidity, similar to the situation during the previous SARS and H1N1 epidemics [64]. A very recent study of healthcare professionals in a tertiary care infectious disease hospital in China revealed a high incidence of anxiety disorders and stress among frontline medical personnel [65], and nurses had a greater incidence of anxiety than doctors. This results in a growing number of healthcare personnel losses, leaving specialized CHD care even more unprotected.

\section{Conclusions}

Children and adults with CHD are an at-risk popula- 
tion in the face of potential SARS-CoV-2 viral infection. The residuals and sequelae not only cause hemodynamic abnormalities, but also systemic changes. The cardiovascular risk factors have been better understood in the general population, but not in the CHD population. This review has shown that several hemodynamic and systemic factors converge in CHD patients, causing serious disorders. It is still unclear whether these risk factors could further increase the lethality and mortality of SARS-CoV-2 virus infection. Perhaps due to the confinement measures still in place in many countries, there are an insufficient number of $\mathrm{CHD}$ patients with SARS-CoV-2 virus infection. Consequently, the current publications only show isolated or associated cases. The pandemic has dramatically transformed the world. One of the consequences has been decreased care of CHD patients, who require constant care and follow up due to their vulnerability. We should understand that the virus has come to stay, and that we must learn to live with it and restart healthcare activities. As long as the effects of the infection are not precisely known and there is no effective treatment for use in CHD patients, we should stay alert and continue with preventive measures in these patients.

\section{Acknowledgement}

To my wife and my daughters D. Lynn and Blanquita. Thanks for your support.

\section{References}

1. Huang C, Wang Y, Li X, Ren L, Zhao J, et al. (2020) Clinical features of patients infected with 2019 novel coronavirus in Wuhan, China. The Lancet 395: 497-506.

2. World Health Organization (2019-nCoV) Novel Coronavirus - Situation report.

3. Aggarwal G, Cheruiyot I, Aggarwal S, Wong J, Lippi G, et al. (2020) Association of cardiovascular disease with coronavirus disease 2019 (COVID-19) severity: A meta-analysis. Curr Probl Cardiol 45: 100617.

4. Xiong TY, Redwood S, Prendergast B, Chen M (2020) Coronaviruses and the cardiovascular system: Acute and long-term implications. Eur Heart J 41: 1798-1800.

5. Zheng YY, Ma YT, Zhang JY, Xie X (2020) COVID-19 and the cardiovascular system. Nat Rev Cardiol 17: 259-260.

6. Sabatino J, Ferrero P, Chessa M, Bianco F, Ciliberti $P$, et al. (2020) COVID-19 and congenital heart disease: Results from a nationwide survey. J Clin Med 9: E1774.

7. Ahluwalia N, Love B, Chan A, Zaidi AN (2020) COVID-19 in an adult with tricuspid atresia S/P Fontan Palliation. JACC Case Rep 2: 1261-1266.

8. Radke RM, Frenzel T, Baumgartner H, Diller GP (2020) Adult congenital heart disease and the COVID-19 pandemic. Heart.

9. Miranda E, Ramírez C, Candela J, Díaz J, Fernández, et al. (2011) Children hospitalized with influenza pneumonia AH1N1/2009 pandemic in the INSN. Rev Peru Med Exp Salud Pública 28: 610-616.

10. Wu S, Wei Z, Greene CM, Yang P, Su J, et al. (2018) Mortality burden from seasonal influenza and 2009 H1N1 pandemic influenza in Beijing, China, 2007-2013. Influenza Other Respir Viruses 12: 88-97.

11. Su S, Wong G, Shi W, Liu J, Lai ACK, et al. (2016) Epidemiology, genetic recombination, and pathogenesis of coronaviruses. Trends Microbiol 24: 490-502.

12. Killerby ME, Biggs HM, Haynes A, Dahl RM, Mustaquim D, et al. (2018) Human coronavirus circulation in the United States 2014-2017. J Clin Virol 101: 52-56.

13. Neher RA, Dyrdak R, Druelle V, Hodcroft EB, Albert J (2020) Potential impact of seasonal forcing on a SARSCoV-2 pandemic. Swiss Med Wkly 150: w20224.

14. Verity R, Okell LC, Dorigatti L, Winskill P, Whittaker C, et al. (2020) Estimates of the severity of coronavirus disease 2019: A model-based analysis. The Lancet Infectus Diseases 20: 669-677.

15. Kissler SM, Tedijanto C, Goldstein E, Grad YH, Lipsitch M (2020) Projecting the transmission dynamics of SARSCoV-2 through the postpandemic period. Science 368: 860868.

16. Anderson RM, Heesterbeek $H$, Klinkenberg D, Hollingsworth T (2020) How will country-based mitigation measures influence the course of the COVID-19 epidemic? Lancet 395: 931-934.

17. Madjid M, Safavi-Naeini P, Solomon SD, Vardeny O (2020) Potential effects of coronaviruses on the cardiovascular system: A Review. JAMA Cardiol.

18. Vardeny O, Madjid M, Solomon SD (2020) Applying the lessons of influenza to covid-19 during a time of uncertainty. Circulation 141: 1667-1669.

19. Koh JWJC, Wong JJM, Sultana R, Wong PPC, Mok YH, et al. (2017) Risk factors for mortality in children with pneumonia admitted to the pediatric intensive care unit. Pediatr Pulmonol 52: 1076-1084.

20. Nyboe C, Olsen MS, Nielsen-Kudsk JE, Johnsen SP, Hjortdal VE (2014) Risk of pneumonia in adults with closed versus unclosed atrial septal defect (from a nationwide cohort study). Am J Cardiol 114: 105-110.

21. Araujo J (2018) Chronic hypoxemic syndrome and congenital heart disease in adults: A multisystemic disorder. J Cardiol \& Cardiovasc Ther 10.

22. Pascall E, Tulloh R (2018) Pulmonary hypertension in congenital heart disease. Future Cardiol 14: 343-353.

23. Brida M, Gatzoulis MA (2018) Pulmonary arterial hypertension in adult congenital heart disease. Heart 104: 15681574.

24. Hoeper MM, Kramer T, Pan Z, Eichstaedt CA, Spiesshoefer J, et al. (2017) Mortality in pulmonary arterial hypertension: Prediction by the 2015 European pulmonary hypertension guidelines risk stratification model. Eur Respir J 50: 1700740.

25. Zhou H, Zhang G, Deng X, Jin B, Qiu Q, et al. (2020) Understanding the current status of patients with pulmonary hypertension during COVID-19 outbreak: A small-scale national survey from China. Pulm Circ 10.

26. Dłużniewska N, Podolec $P$, Skubera $M$, Smaś-Suska $M$, Pająk J, et al. (2018) Long-term follow-up in adults after tetralogy of Fallot repair. Cardiovasc Ultrasound 16: 28.

27. O'Byrne ML, Glatz AC, Mercer-Rosa L, Gillespie MJ, Dori $Y$, et al. (2015) Trends in pulmonary valve replacement in children and adults with tetralogy of fallot. Am J Cardiol 115: 118-124. 
28. Loomba RS, Buelow MW, Aggarwal S, Arora RR, Kovach $\mathrm{J}$, et al. (2017) Arrhythmias in adults with congenital heart disease: What are risk factors for specific arrhythmias? Pacing Clin Electrophysiol 40: 353-361.

29. Araujo JJ (2018) Commentary on the New 2018 AHA/ACC Guideline for the management of adults with congenital heart disease. CPQ Cardiology 1: 1-10.

30. Attenhofer Jost $\mathrm{CH}$, Connolly HM, Dearani JA, Edwards WD, Danielson GK (2007) Ebstein's anomaly. Circulation 115: 277-285.

31. Walsh EP, Cecchin F (2007) Arrhythmias in adult patients with congenital heart disease. Circulation 115: 534-545.

32. Wang D, Hu B, Hu C, Zhu F, Liu X, et al. (2020) Clinical characteristics of 138 hospitalized patients with 2019 novel coronavirus-infected pneumonia in Wuhan, China. JAMA 323: 1061-1069.

33. Vandael E, Vandenberk B, Vandenberghe J, Willems R, Foulon V (2017) Risk factors for QTc-prolongation: Systematic review of the evidence. Int J Clin Pharm 39: 16-25.

34. Heemskerk CPM, Pereboom M, van Stralen K, Berger FA, van den Bemt PMLA, et al. (2018) Risk factors for QTc interval prolongation. Eur J Clin Pharmacol 74: 183-191.

35. Chvilicek JP, Hurlbert BJ, Hill GE (1995) Diuretic-induced hypokalaemia inducing torsades de pointes. Can J Anaesth 42: 1137-1139.

36. Mehra MR, Desai SS, Kuy SR, Henry TD, Patel AN (2020) Retraction: Cardiovascular disease, drug therapy, and mortality in Covid-19. N Engl J Med 382: 2582.

37. Mehra MR, Ruschitzka F, Patel AN (2020) Retraction-Hydroxychloroquine or chloroquine with or without a macrolide for treatment of COVID-19: A multinational registry analysis. Lancet 395: 1820.

38. Perloff JK (2008) Cyanotic congenital heart disease: A multisystem disorder. In: Perloff JK, Child JS, Aboulhosn $J$, Congenital Heart Disease in Adults. ( $3^{\text {rd }}$ edn), Elsevier, Philadelphia, USA, 265-289.

39. http://www.siacardio.com/novedades/covid-19/recomendaciones-del-consejo-de-cardiopatias-congenitas-del-adultopara-el-cuidado-y-atencion-de-pacientes-frente-a-la-pandemia-covid-19/

40. Couzin-Frankel J (2020) The mystery of the pandemic's 'happy hypoxia'. Science 368: 455-456.

41. Xie J, Covassin N, Fan Z, Singh P, Gao W, et al. (2020) Association between hypoxemia and mortality in patients with COVID-19. Mayo Clin Proc 95: 1138-1147.

42. Kashani KB (2020) Hypoxia in COVID-19: Sign of severity or cause for poor outcomes. Mayo Clin Proc 95: 1094-1096.

43. Zhou F, Yu T, Du R, Fan G, Liu Y, et al. (2020) Clinical course and risk factors for mortality of adult inpatients with COVID-19 in Wuhan, China: A retrospective cohort study. Lancet 395: 1054-1062.

44. Soria F, Romero A, Vicente T (2020) Controversy regarding ACE inhibitors/ARBs in Covid-19. Rev Esp Cardiol 73: 516.

45. Kauw D, Woudstra O, van Engelen K, Meijboom FJ, Mulder BJM, et al. (2020) 22q11.2 deletion syndrome is associated with increased mortality in adults with tetralogy of Fallot and pulmonary atresia with ventricular septal defect. Int $J$ Cardiol 306: 56-60.

46. Yilmaz E, Ustundag B, Sen $Y$, Kurt AN, Dogan $Y$, et al. (2007) The levels of Ghrelin, TNF- $\alpha$, and IL-6 in children with cyanotic and acyanotic congenital heart disease. Mediators Inflamm 2007: 32403.
47. Zhang Q, Wang $H$, Xue J, Wu D (2020) Associations between IL-6 Variations and congenital heart disease incidence among Chinese Han People. Med Sci Monit 26: e921032.

48. Riphagen S, Gomez X, Gonzalez-Martinez C, Wilkinson N, Theocharis $P(2020)$ Hyperinflammatory shock in children during COVID-19 pandemic. Lancet 395: 1607-1608.

49. Esper F, Shapiro ED, Weibel C, Ferguson D, Landry ML, et al. (2005) Association between a novel human coronavirus and Kawasaki disease. J Infect Dis 191: 499-502.

50. Cheung EW, Zachariah P, Gorelik M, Boneparth A, Kernie SG, et al. (2020) Multisystem inflammatory syndrome related to COVID-19 in previously healthy children and adolescents in New York City. JAMA e2010374.

51. Verdoni L, Mazza A, Gervasoni A, Martelli L, Ruggeri M, et al. (2020) An outbreak of severe Kawasaki-like disease at the Italian epicentre of the SARS-CoV-2 epidemic: An observational cohort study. Lancet 395: 1771-1778.

52. Henriksson P, Värendh G, Lundström NR (1979) Haemostatic defects in cyanotic congenital heart disease. Br Heart J 41: 23-27.

53. Chamanian S, Maleki M, Rohani A, Amini M (2015) Hematologic abnormalities in cyanotic congenital heart disease patients. J Cardiothorac Med 3: 270-272.

54. Atri D, Siddiqi HK, Lang J, Nauffal V, Morrow DA, et al. (2020) COVID-19 for the cardiologist: A current review of the virology, clinical epidemiology, cardiac and other clinical manifestations and potential therapeutic strategies. JACC Basic TransI Sci 5: 518-536.

55. Rathgeber SL, Guttman OR, Lee AF, Voss C, Hemphill NM, et al. (2020) Fontan-associated liver disease: Spectrum of disease in children and adolescents. J Am Heart Assoc 9: e012529.

56. Guan W, Ni Z, Hu Y, Liang W, Ou C, et al. (2020) Clinical characteristics of coronavirus disease 2019 in China. $N$ Engl J Med 382: 1708-1720.

57. Shi S, Qin M, Shen B, Cai Y, Liu T, et al. (2020) Association of cardiac injury with mortality in hospitalized patients with COVID-19 in Wuhan, China. JAMA Cardiol 5: 802-810.

58. Cheng Y, Luo R, Wang K, Zhang M, Wang Z, et al. (2020) Kidney disease is associated with in-hospital death of patients with COVID-19. Kidney Int 97: 829-838.

59. Sanders SP, Geva T (2018) Classifying heterotaxy syndrome: Time for a new approach. Circ Cardiovasc Imaging 11: e007490.

60 . Kothari SS (2014) Non-cardiac issues in patients with heterotaxy syndrome. Ann Pediatr Cardiol 7: 187-192.

61. https://www.mscbs.gob.es

62. Blustein DL, Duffy R, Ferreira JA, Cohen-Scali V, Cinamon $\mathrm{R}$, et al. (2020) Unemployment in the time of COVID-19: A research agenda. J Vocat Behav 119: 103436.

63. DeFilippis EM, Stefanescu Schmidt AC, Reza N (2020) Adapting the educational environment for cardiovascular fellows-in-training during the COVID-19 Pandemic. J Am Coll Cardiol 75: 2630-2634.

64. Pfefferbaum B, Schonfeld D, Flynn BW, Norwood AE, Dodgen D, et al. (2012) The H1N1 crisis: A case study of the integration of mental and behavioral health in public health crises. Disaster Med Public Health Prep 6: 67-71.

65. Tsamakis K, Rizos E, J Manolis A, Chaidou S, Kympouropoulos S, et al. (2020) COVID-19 pandemic and its impact on mental health of healthcare professionals. Exp Ther Med 19: 3451-3453. 